\title{
PROTEIN SIGNAL TRANSDUCERS AND ACTIVATORS TRANSCRIPTION (STAT) AS GROWTH PROMOTER
}

\author{
Anwar Ma'ruf ${ }^{1,2}$, Ngakan Made RW', \\ M. Sukmanadi, Ratna Damayanti ${ }^{2}$
}

\author{
${ }^{1}$ Postgraduate School, Universitas Airlangga Surabaya-Indonesia, \\ ${ }^{2}$ Department of Basic Veterinary Medicine, Faculty of Veterinary Medicine Universitas Airlangga Surabaya-Indonesia
}

\begin{abstract}
The purpose of this study was to determine the amino acid composition of proteins STAT 1 and STAT 3 existing in the liver tissue of broiler chickens that are experiencing growth due to increased growth hormone $(G H)$. Liver tissue samples of broilers isolated from broiler chickens reared for 21 days were examined using SDS Page, followed by Western Blott and MALDI-TOP. Results of Western Blot revealed that STAT protein had molecular weight of $59.3 \mathrm{kDa}$ with the first amino acid composition 1, mtqwyqlqqu dskflequhq lyddsfpmei rqylaqwlen qdwehaannv sfatvlfhdl. 61 lsnqmevggv qntmtgmldk qkeldakvka vknsvtdveq diktledvqd eydfkhktfq, 121 lsqlddqfsr fliennfllq hnirkskrnl qdnfqedpih mamiihnclk eerkilnsaq, and STAT 3 59.4kDa by arrangement amino acids 1, maqwnqlqql dtryleqlhq lysdsfpmel rqflapwies qdwayaanke shatlvfhnl, 121 taaqqggqat hptaavvtek qqmleqhlqd vrkrvqdleq kmkvvenlqd dfdfnyktlk, 181 sqgdmqding nnqsvtrqkm qqleqmital dqmrrgivse lagllsamey vqkmladeel, 241 adwkrrqqia ciggppnicl drlenwitsl aesqlqtrqq ikkleelqqk vsykgdpivq, 301 hrpmleeriv elfrnlmksa fvverqpcmp mhpdrplvik tgvqfttkvr llvkfpelny and 361 qlkikvcidk dsgdvaalrg srkfnilgtn tkvmnmeesn ngslsaefkh ltlreqrcgn.
\end{abstract}

Keywords: STAT 1, STAT 3, growth, broiler

\section{INTRODUCTION}

Growth hormone has important meaning in regulating body growth and metabolism. GH metabolic effects occur when GH receptors associate with and activate tyrosine kinases. The GH bond with its receptor may activate Janus Kinase 2 (JAK 2) and further phosphorylate tyrosine in the JAK-2 GH-receptor complex. This tyrosine then forms the binding site for a number of signaling proteins, such as signal transducers and activators of transcription (STAT) to incite the effect of growth. STAT proteins that play a role in providing growth signaling are STAT 1, STAT 3, STAT 5a and STAT B. STAT proteins play a significant role in regulating metabolic and growth effects.
Increased growth in animal husbandry has great implications and appeal in the poultry field. However STAT protein signaling and its expression pattern in broilers during growth period has not been indentified clearly. Therefore, knowledge on molecular weight and the arrangement of amino acids of STAT signaling protein in broilers during growth period due to increased GH can be used to make synthetic STAT protein to spur the growth of the broilers.

Until recently, the only known identification of amino acid STAT 5a protein composition was ciggppkvmnmeesn, and STAT 5b protein was datnilvspvylypdip (Anwar et al., 2013). Therefore, we need further studies to identify the composition of amino acid protein STAT 1 and STAT 3 to be used as the basis of producing synthetic STAT protein to boost the growth of the broilers.

\section{MATERIALS AND METHODS}

Chickens were placed in a batteried cage with a capacity of one chicken per cage with twice daily feed at 6:00am and 6:00pm with the amount of $10 \%$ smaller than the standard. At the age of 21 days, the chickens were sacrificed to be sampled by taking their hepatic tissue for the following tests: (1) isolation of signaling proteins STAT 1 and STAT 3 from broiler liver tissue; (2) analysis of STAT 1 and STAT 3 protein signaling from broiler liver tissue; (3) identification of molecular weight of STAT 1 and STAT 3 signaling proteins by blotting method, the Western blot technique, using electrophoretically elaborated protein from polyacrylamide gel; and (4) identification of amino acids by MALDI-TOF method.

\section{RESULTS AND DISCUSSION}

\section{SDS Page of STAT protein from broiler liver}

The result of SDS-PAGE STAT protein on broiler liver tissue showed the presence of STAT protein, as in Figure 1. 


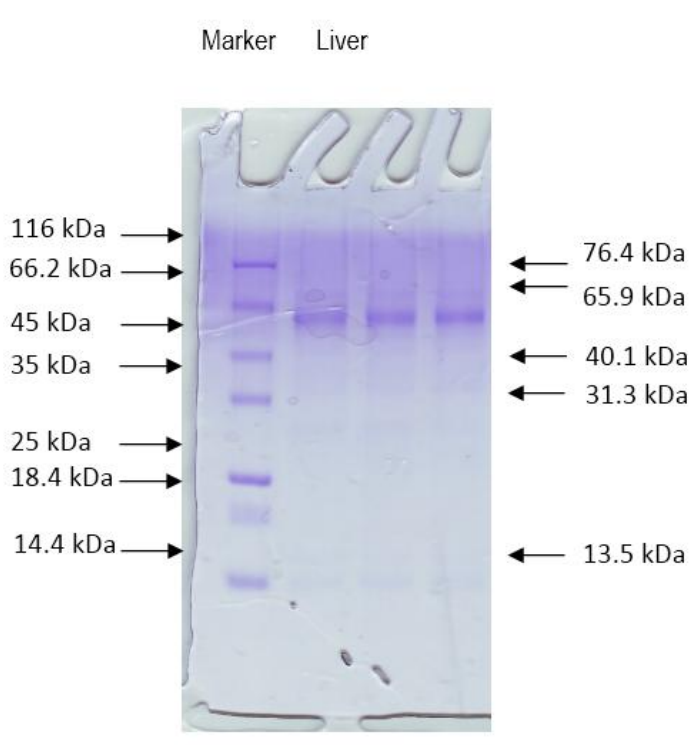

Figure 1. SDS Page STAT protein from the liver tissue

The SDS-PAGE results showed five visible protein bands between the $116 \mathrm{kDa}$ marker and $14.4 \mathrm{kDa}$ on the liver tissue.

From the calculations (Table 5.1), the protein bands formed between $116 \mathrm{kDa}$ markers with $14.4 \mathrm{kDa}$ had molecular weights of $76.4 \mathrm{kDa}, 65.9 \mathrm{kDa}, 40.1 \mathrm{kDa}$, $31.3 \mathrm{kDa}$ and $13.5 \mathrm{kDa}$. The protein bands formed on the hepatic tissue were apparently very clear at the molecular weight of 65.9kDa. Results of SDS-PAGE liver tissue protein showed the most obvious protein bands were between the $66.2 \mathrm{kDa}$ marker, with $45 \mathrm{kDa}$, the suspected proteins STAT 1 and STAT 3. The proteins of the SDS-PAGE results could not be ascertained yet as to whether they were STAT 1 or STAT 3 proteins. To prove that the formation of protein bands were STAT 1 and STAT 3 proteins it was necessary to check with Western blot.

\section{Western Blot of STAT 1 protein}

The result of Western blot STAT protein on hepatic tissue showed the presence of STAT 1 protein with a molecular weight of $59.3 \mathrm{kDa}$, as in Figure 2.

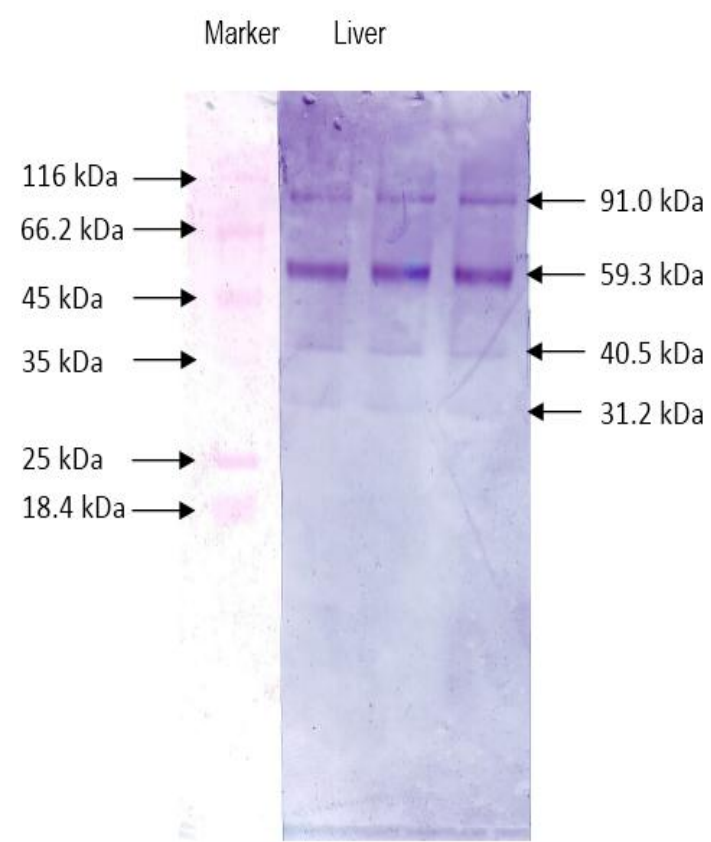

Figure 2. Western Blot of STAT-1 protein from the liver tissue

To verify that the result of protein analysis with SDSPAGE was STAT 1 protein, then the Western blot was done using rabbit polyclonal antibody STAT 1 Ab-1 (Labvision). In Figure 3, it appears that one protein band is most apparent between the $66.2 \mathrm{kDa}$ marker, with $44 \mathrm{kDa}$, a protein formed on the liver tissue. The protein bands formed between $116 \mathrm{kDa}$ markers with $66.2 \mathrm{kDa}, 45 \mathrm{kDa}$ with $35 \mathrm{kDa}$ and $35 \mathrm{kDa}$ with $2 \mathrm{kDa}$ are not very clear.

After calculation (Table 5.2), the formation of protein bands between markers $66.2 \mathrm{kDa}$ with $44 \mathrm{kDa}$ turned out to have a molecular weight of $59.3 \mathrm{kDa}$. This suggests that the SDS-PAGE-produced protein tested with Western blot was a STAT 1 protein in broiler during growth period with a molecular weight of $59.3 \mathrm{kDa}$. The formation of a very clear $56.3 \mathrm{kDa}$ band of protein bands was due to the occurrence of a binding between the SDS-PAGE STAT 1 protein with rabbit polyclonal antibody STAT 1.

\section{Western Blot Protein STAT 3}

The result of Western blot protein STAT on hepatic tissue showed the presence of STAT 3 protein with a molecular weight of $59.4 \mathrm{kDa}$, as in Figure 3. 


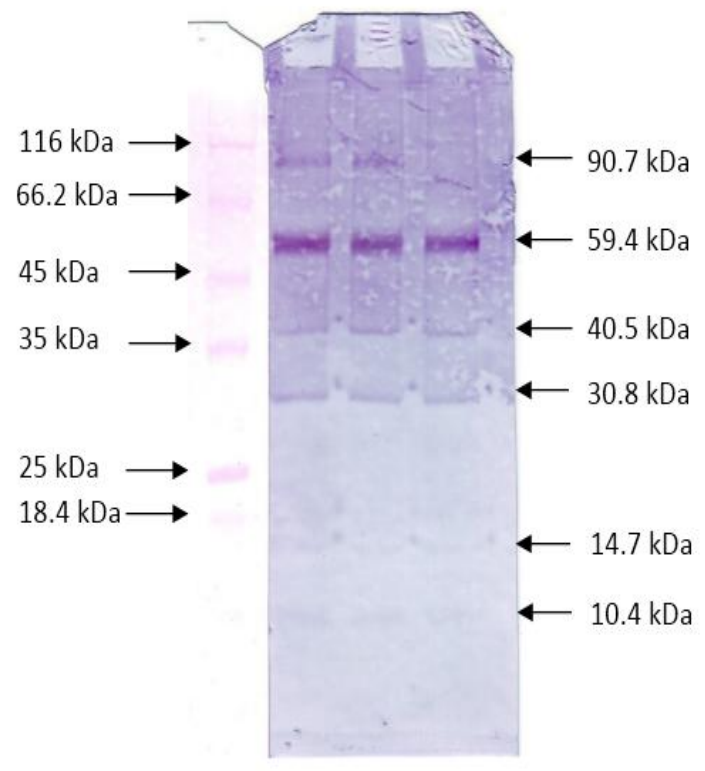

Figure 3. Western Blot of STAT-3 protein from the liver tissue

To ensure that protein analysis results with SDSPAGE was a STAT 3 protein, the Western blot was performed using rabbit polyclonal antibody STAT 3 Ab-1 (Labvision). Figure 5.4 shows the formation of one of the clearest protein bands between the $66.2 \mathrm{kDa}$ marker and 44kDa; both proteins formed on adipose, muscle and liver tissue. The protein bands formed between $116 \mathrm{kDa}$ markers with $66.2 \mathrm{kDa}, 45 \mathrm{kDa}$ with $35 \mathrm{kDa}$ and $35 \mathrm{kDa}$ with $25 \mathrm{kDa}$ are not very clear

After calculation (Table 5.3), the protein band formed between the $66.2 \mathrm{kDa}$ marker and $44 \mathrm{kDa}$ has a molecular weight of $59.4 \mathrm{kDa}$. This suggests that the SDS-PAGE protein tested with Western blot is a STAT 3 protein from broilers during growth period with molecular weight of $59.4 \mathrm{kDa}$. The protein band with a molecular weight of $59.4 \mathrm{kDa}$ was very clearly formed due to the binding between STAT 3 protein SDS-PAGE results with rabbit polyclonal antibody STAT 3.

\section{Amino acid of STAT 1 and 3 proteins}

Results of examination using MALDI-TOP method showed that STAT 1 protein with molecular weight of $59.3 \mathrm{kDa}$ had amino acid 1 arrangement of mtqwyqlqql dskfleqvhq lyddsfpmei rqylaqwlen qdwehaannv sfatvlfhdl, 61 lsnqmevggv qntmtgmldk qkeldakvka vknsvtdveq diktledvqd eydfkhktfq, 121 lsqlddqfsr fliennfllq hnirkskrnl qdnfqedpih mamiihnclk eerkilnsaq, and STAT 3 as much as $59.4 \mathrm{kDa}$ with amino acid 1 arrangement maqwnqlqql dtryleqlhq lysdsfpmel rqflapwies qdwayaanke shatlvfhnl, 121 taaqqggqat hptaavvtek qqmleqhlqd vrkrvqdleq kmkvvenlqd dfdfnyktlk, 181 sqgdmqdlng nnqsvtrqkm qqleqmital dqmrrgivse lagllsamey vqkmladeel, 241 adwkrrqqia ciggppnicl drlenwitsl aesqlqtrqq ikkleelqqk vsykgdpivq 301 hrpmleeriv elfrnlmksa fvverqpemp mhpdrplvik tgvqfttkvr llvkfpelny, and 361 qlkikvcidk dsgdvaalrg srkfnilgtn tkvmnmeesn ngslsaefkh ltlreqrcgn.

Growth hormone plays a role in regulating body growth and composition (Foster, 1998). Growth hormone apparently has biological effects that are influenced by insulin-like growth factor I (IGF-I) in enhancing the growth of skeletal muscle (Younken, 2000). Provision of in vivo growth factor in broilers led to an increase in growth rate and muscle mass by $15 \%$ and required $6.5 \%$ less feed than normal feed. This increase in growth has major implications and appeal in the field of poultry. However, to date, expression patterns of growth factor genes during growth period have not been clearly identified (Killefer, 2000).

STAT proteins play an important role in the regulation of gene transcription by $\mathrm{GH}$ and other cytokines that activate Janus Kinase (JAK). STAT proteins, originally identified in the signaling pathway of interferon (IFN) (Darnell et al., 1994), is a cytoplasmic factor containing domain $\mathrm{SH}-2$. In tyrosyl phosphorylation, frequently through the cascade initiated by JAK kinases, STAT protein cytoplasm forms a complex with other STAT protein through the interaction of tyrosine which is phosphorylated on the domain of $\mathrm{SH}-2$, and holds translocations leading to the nucleus, binds to DNA and subsequently activates transcription of the gene target (Ihle, 1996).

Growth hormone is known to activate STATs $1,3,5 \mathrm{a}$ and 5b. Phosphorylation of GH-dependent STATS 1, 3 , 5a and $5 \mathrm{~b}$ tyrosyl is found in 3T3-F442A fibroblasts, liver of rats with hypophysectomy, liver cell cultures and in various systems' overexpression. Phosphorylation of STATS 5a and 5b tyrosyl is also found in IM-9 human cells and muscles of the liver and skeletal muscle of normal mice (Smit et al., 1999).

STAT1, also called P91, is identified as a member of the factor 3 gene complex that is stimulated by IFNgamma (FU, 1992). Analysis of signaling $\mathrm{GH}$ in JAK2 deficient cells and cells undergoing mutations in expressing $\mathrm{GH}$ receptor indicates that $\mathrm{GH}$ dependent activation of STATS 1, 3, 5a and 5b requires activation of JAK2 (Smit et al., 1997). This is consistent with the finding that JAKs activation is required for STAT activation (Muller et al., 1993). JAK1 or JAK2 actively overexpressed in COS cells will stimulate the binding of STAT1 to DNA (Silvennoinen, 1993).

Indirect research has shown that GH stimulates the phosphorylation of STATs 1, 3 and 5 in serine or 
threonine in the liver. This phosphorylation will increase DNA binding of STAT1, DNA STAT3 and substantially alter DNA binding of STAT5 (Ram et.al., 1996). STAT 1, 3, and 5a contain conserved consensus sequences for phosphorylation, the MAP kinase. Initial studies have shown that MAP kinase is responsible for serial phosphorylation of STAT1, STAT3 and STAT 5a. Whereas, in STAT 5b, since it does not contain conserved consensus sequences, the phosphorylation is performed by kinases other than MAP kinase. Proteins STAT 1, 3, 5a and 5b also contain protein kinase $\mathrm{C}$ and casein kinase for phosphorylation process. This suggests that double signaling pathways can converge in STAT proteins for transcriptional activation by $\mathrm{GH}$.

\section{CONCLUSIONS OR IMPLICATIONS}

1. STAT-1 protein is 1 mtqwyqlqql dskfleqvhq lyddsfpmei rqylaqwlen qdwehaannv sfatvlfhdl, 61 lsnqmevggv qntmtgmldk qkeldakvka vknsvtdveq diktledvqd eydfkhktfq, 121 lsqlddqfsr fliennfllq hnirkskrnl qdnfqedpih mamiihnclk eerkilnsaq.

2. STAT-3 1 protein is maqwnqlqql dtryleqlhq lysdsfpmel rqflapwies qdwayaanke shatlvfhnl, 121 taaqqggqat hptaavvtek qqmleqhlqd vrkrvqdleq kmkvvenlqd dfdfnyktlk, 181 sqgdmqdlng nnqsvtrqkm qqleqmital dqmrrgivse lagllsamey vqkmladeel, 241 adwkrrqqia ciggppnicl drlenwitsl aesqlqtrqq ikkleelqqk vsykgdpivq, 301 hrpmleeriv elfrnlmksa fvverqpcmp mhpdrplvik tgvqfttkvr llvkfpelny, 361 qlkikvcidk dsgdvaalrg srkfnilgtn tkvmnmeesn ngslsaefkh ltlreqrcgn.

3. By knowing the arrangement of amino acids, it can be employed as the basis for making STAT synthetic protein which is expected to be used to prolong the action or effect of growth hormone so that it can enhance the growth of livestock.

\section{REFERENCES}

Darnell J, Lodish H, Baltimora D., 1990. Molecular cell biology. 2nd Edition, New York: Scientific America Books, p 715

Foster DN, Froudman JA, Harmon SA, Foster LK., 1998. Baculovirus-mediated expression of chicken GH. Tektran p 14

Ihle JN., 1996. Cell $84: 331-334$

Kaiya H, Van Der Geyten S, Kojima M, Hosoda H, Kitajima Y, Matsumoto M, Geeliissen S, Darras VM and Kanagawa K. 2001. Chicken Ghrelin : purification cDNA cloning, and biological activity. Endocrinology $143: 3463$

Killefer K, Kenny PB., 2000. Anim Vet Sci 1877.

Ram PA and Waxman DJ., 1999. J. Biol. Chem. 274:35553-35561
Ram PA, Park SH, Choi HK and Waxman DJ., 1996. J. Biol. Chem. 271:5929-5940

Smit LS, Meyer DJ, Argetsinger LS et al., 1999 Handbooks of Physiology. Oxford University Press : New York, pp445-480.

Younken RV, Zaou Y, Wang X et al., 2000. J Endocrinol 166:620-690 